\title{
Trajectoires de vie et prises de risques chez les femmes usagères de drogues
}

\author{
Marie JAUfFRET-ROUSTIDE ${ }^{1,2}$, Lila OUdAYA ${ }^{1}$, Marc RONDY ${ }^{2}$, Yao KUDAWU ${ }^{1}$, \\ Yann Le Strat ${ }^{1}$, Elisabeth CouturieR ${ }^{1}$, Julien EMMANUELLI ${ }^{1}$, Jean-Claude Desenclos ${ }^{1}$
}

\section{Résumé}

En France, la population des usagers de drogues est majoritairement masculine. Parmi ceux-ci, les profils et les pratiques des femmes sont mal connus. Entre 2004 et 2007, l'enquête Anrs-Coquelicot, alliant épidémiologie et socio-anthropologie, a permis de décrire les profils des usagers de drogues et d'analyser les situations à risque vis-à-vis du $\mathrm{VIH}$ et du VHC, en prenant en compte la problématique du genre. Les entretiens montrent que les femmes ont fréquemment connu des événements marquants durant l'enfance et l'adolescence (violences). C'est généralement dans ce contexte qu'elles s'initient aux drogues par le biais de rencontres amoureuses. Elles adoptent globalement plus de comportements à risque que les hommes et ont davantage recours à la prostitution.

Ainsi, ces femmes ont des trajectoires encore plus chaotiques que celles des hommes. Elles sont confrontées à une double fragilité vis-à-vis du VIH et du VHC liée au contexte de leur consommation de drogues et de leur sexualité.

Mots clés: usage de drogues, vulnérabilité, épidémiologie, socioanthropologie, comportements à risque.

En France, les données disponibles sur les usagers de drogues portent sur une population constituée majoritairement d'hommes, et ne comprend généralement qu'un quart de femmes. Cette répartition entre hommes et femmes est issue d'études portant sur la population spécifique des usagers de drogues ayant recours à une prise en charge pour leur problème d'addiction, soit dans les dispositifs spécialisés, soit chez les médecins généralistes. Ces études ne prennent donc pas en compte les populations d'usagers de drogues peu visibles, c'est-à-dire ne fréquentant pas les dispositifs pour lesquels nous ne disposons pas de la répartition hommes/femmes. Ainsi, en France, la population des usagères de drogues est mal connue car la plupart des recherches effectuées dans ce champ ne prennent pas en compte la question du genre. Quand les femmes sont étudiées, c'est à travers leur identité de mère et des risques liés à la prise de toxiques durant la grossesse [1]. Les articles qui s'intéressent

\footnotetext{
Institut national de veille sanitaire, Saint-Maurice.

Centre de recherches psychotropes, santé mentale et société (unité Inserm 811, UMR 8136 CNRS, Université Paris V - Descartes.
}

spécifiquement aux profils et aux prises de risques spécifiques chez les femmes usagères de drogues sont rares et anciens [2].

Dans la littérature internationale, en revanche, les publications relatives aux usagères de drogues sont plus nombreuses. Cette population y est souvent appréhendée à travers les consommatrices de crack [3] et est donc décrite comme particulièrement précaire, et marquée par un cumul de handicaps sociaux et psychologiques. Les femmes usagères de drogues y sont également étudiées à travers le prisme de leur vulnérabilité vis-à-vis de I'infection à VIH [4-6]

II semblait donc intéressant de présenter des données françaises sur la question des femmes usagères de drogues et d'analyser leurs éventuelles spécificités, en termes de trajectoires de vie et de prises de risque liées à l'usage de drogues et à la sexualité, à partir des données issues de l'enquête Anrs-Coquelicot, menée par l'Institut national de veille sanitaire entre 2004 et $2007^{3}$.

\section{Matériel et méthodes}

\section{Épidémiologie et socio-anthropologie, deux approches qui se complètent}

Dans cet article, les principaux résultats sont issus de l'approche épidémiologique (quantitative) de l'enquête Anrs-Coquelicot, mais quelques résultats de l'étude socio-anthropologique (qualitative) sont également présentés.

La partie qualitative de l'enquête n'est pas ici analysée de manière autonome, mais vient en complément de l'approche épidémiologique, afin d'illustrer par des trajectoires biographiques certains éléments mis en évidence ou afin de préciser certains éléments spécifiques aux parcours des femmes que la partie quantitative n'a pas explorés. Ainsi, les entretiens ont donné la possibilité de prendre en compte la temporalité inhérente aux trajectoires, d'appréhender le contexte dans lequel s'inscrivaient

L'étude Anrs-Coquelicot est une enquête de séroprévalence du VIH et du VHC chez les usagers de drogues, réalisée fin 2004 par l'Institut national de veille sanitaire, en collaboration avec l'Institut national d'études dèmographiques (Ined) et le Centre national de référence du VIH de Tours pour le volet épidémiologique (quantitatif) de l'étude,et en collaboration avec le Centre de recherches psychotropes, santé mentale et société (Inserm/CNRS/Paris V) pour le volet socio-anthropologique (qualitatif) réalisé entre 2005 et 2007. L'ensemble de l'étude a été soutenu par l'Agence nationale de recherches sur le sida et les hépatites virales (Anrs). 
les pratiques et de saisir les logiques sociales qui les soustendent. L'approche qualitative a permis de partir de l'expérience intime des usagères de drogues et d'aborder certaines « questions sensibles " sur les ruptures de vie, en particulier les violences subies durant l'enfance, l'adolescence ou l'âge adulte, et la prostitution. Ces thématiques, évoquées brièvement dans le questionnaire épidémiologique, ont pu être développées dans le cadre des entretiens.

Ainsi, l'approche socio-anthropologique a permis d'affiner, voire d'interroger certains résultats quantitatifs liés aux prises de risque mis en évidence lors de l'analyse épidémiologique, tels que la phase d'initiation à la consommation de drogues, les contextes de partage du matériel d'injection, la vie affective et sexuelle. Les personnes interrogées ont été amenées à décrire en détail les processus d'initiation et d'apprentissage par les pairs. Leurs pratiques ont alors été réinscrites dans des relations d'interdépendance avec le contexte social. Chaque usager a été réintégré dans les différents réseaux sociaux auxquels il participait, afin d'appréhender, par exemple, la dimension de la précarité sociale et relationnelle ou d'analyser les prises de risque spécifiques dans le couple, en prenant en compte la dynamique sociale des relations. L'utilisation de la technique des récits de vie nous a donné la possibilité d'interroger l'usager de drogue sur la place qu'occupe le risque aux différents moments de sa trajectoire, d'étudier les espaces dans lesquels il consomme des produits psychoactifs, et d'explorer ses liens de proximité avec ses partenaires de partage. Les récits des trajectoires biographiques des individus ont permis de repérer les points de rupture propices à la prise de risques et d'aller au-delà du caractère instantané des données recueillies dans le volet épidémiologique.

\section{Méthodologie du volet séro-épidémiologique}

Le volet épidémiologique de l'étude Anrs-Coquelicot est une enquête descriptive transversale auprès d'un échantillon aléatoire d'usagers de drogues. Les sujets inclus sont des volontaires ayant injecté et/ou sniffé au moins une fois dans leur vie. 1472 sujets ont été inclus entre septembre et décembre 2004. Cette enquête est multivilles (Lille, Strasbourg, Paris, Bordeaux, Marseille) et le recrutement des usagers de drogues s'est effectué dans 101 services issus de la chaine thérapeutique spécifique (centres de soins spécialisés pour toxicomanes, centres de post-cure, appartements thérapeutiques, sleep-in, boutiques, programmes d'échange de seringues, équipes de rue) et dans 36 cabinets de médecins généralistes prescripteurs de traitements de substitution aux opiacés.

Un plan de sondage stratifié (villes et services/médecins) a été mis en œuvre. Pour chaque ville, une base de sondage des services par demi-journée d'ouverture a été constituée. Des couples (services-demi-journée) basés sur les files actives (constituées de sujets usagers de drogues) déclarées par les services ont été tirés au sort par un sondage aléatoire simple sans remise, permettant de constituer un calendrier des visites au cours de la période d'enquête. Au sein de chaque service, les usagers de drogues étaient tirés au sort de manière aléatoire, à l'exception des hébergements où tous étaient interrogés. Pour ce qui concerne les médecins, un sondage en grappe a été effectué dans chaque ville. Un sondage aléatoire simple des médecins prescripteurs, avec stratification sur le volume de prescription de traitements de substitution (gros/moyen prescripteurs), a été mis en place. Puis tous les usagers de drogues ont été enquêtés chez les médecins tirés au sort.

Le recueil des données consiste en un questionnaire sociocomportemental administré à l'usager de drogues par un enquêteur professionnel et en un autoprélèvement de sang au doigt. Un questionnaire d'une durée de 30-40 minutes était administré en face-à-face par des enquêteurs dans les structures spécialisées et par des médecins dans les cabinets de médecine de ville. Le questionnaire portait sur les dimensions suivantes: situation sociodémographique des individus, représentation de leur état de santé, accès aux soins et à la prévention, situations et comportements vis-à-vis du VIH, du VHC et du VHB, pratiques liées à la consommation de produits injectés, snifés, inhalés, connaissances vis-à-vis des risques d'exposition virale (connaissance des modes de transmission, perception de ces maladies et de leurs traitements), pratiques à risque liées à la consommation et la sexualité. Afin d'estimer la séroprévalence des anticorps contre le VIH et le VHC et de la comparer à celle estimée de manière déclarative, un autoprélèvement de sang au doigt était systématiquement proposé à l'usager de drogues. Le prélèvement était réalisé par le sujet lui-même, avec le matériel (buvard, microlancette) remis par l'enquêteur. L'analyse des buvards a été réalisée par le Centre national de référence du VIH de Tours à partir de tests Elisa pour le VIH et le VHC.

\section{Méthodologie du volet socio-anthropologique}

Une approche socio-anthropologique complète l'approche épidémiologique, en amont, lors de la construction des hypothèses, du questionnaire et de la préparation du terrain, mais également dans le cadre d'un volet socio-anthropologique à part entière. Ce volet [7] avait pour objectifs d'accéder à des populations difficiles d'accès, d'approfondir mais aussi d'interroger certains résultats quantitatifs. La méthodologie a consisté en des entretiens semidirectifs (87) et des observations ethnographiques sur des lieux de consommation et de deal.

La population de l'étude socio-anthropologique n'étant pas connue précisément au départ, l'échantillon a été constitué de manière progressive par une méthode dite boule de neige. Plusieurs sous-groupes d'usagers ont été contactés et étudiés : des jeunes (moins de 30 ans), des femmes, des étudiants, et des consommateurs très marginalisés. L'apport d'une approche socio-anthropologique est ici de faire émerger des réseaux d'individus qui ne sont pas habituellement captés dans les études 
épidémiologiques et qui constituent une partie de la population cible.

Ces entretiens semi-directifs se sont déroulés à partir d'une grille commune évoquant la trajectoire personnelle de l'usager de drogues et détaillant plus particulièrement les comportements d'usage et les pratiques à risque. Durant ces entretiens, l'enquêteur partait d'une consigne commune et s'efforçait ensuite d'intégrer l'ensemble des thématiques présentes dans une grille d'entretien préétablie, en laissant toujours à la personne la possibilité d'évoquer des thèmes non prévus. Contrairement au questionnaire, le guide d'entretien évolue en fonction de la dynamique de chaque situation d'enquête. Dans l'analyse, les façons de parler et l'interaction entre l'enquêteur et l'enquêté sont à prendre en compte tout autant que le contenu de ce qui est dit. La plupart des entretiens effectués dans l'étude Anrs-Coquelicot s'apparentent, en fait, à des récits de vie car chaque entretien est centré sur la trajectoire de l'usager de drogues et comporte une dimension rétrospective et réflexive importante.

L'analyse des entretiens a été réalisée à partir du logiciel NVivo permettant de gérer plus facilement une base de données discursives conséquente. L'utilisation de ce logiciel laisse toute sa place au chercheur pour le choix des thèmes relatifs à la codification des données et pour l'interprétation des données. Une lecture attentive des entretiens associée aux axes problématiques de la recherche a permis l'établissement d'une grille de codification (arbre), constituée par des unités de sens (nœuds), processus nécessaire à l'indexation des entretiens dans NVivo. Dans le cadre de ce chapitre, une première étape d'analyse de contenu thématique a été réalisée dans une logique inductive, en partant des données empiriques. II s'agissait ici de partir des expériences vécues par les personnes en s'efforçant de saisir le sens qu'elles attribuent à leurs actions et en observant la manière dont elles racontent et interprètent leurs trajectoires personnelles. Des analyses plus approfondies à partir de la création d'attributs sont actuellement en cours dans le cadre de l'utilisation du logiciel NVivo. Ces attributs ont pour objectif la discrimination des données et permettront de saisir plus finement les logiques sociales qui sous-tendent les actions des usagers de drogues. Un raisonnement de type comparatif permettant la construction de typologies sera enfin appliqué au corpus d'entretiens, sans prétendre à la généralisation et en veillant à conserver les singularités des trajectoires biographiques de chaque usager de drogues.

\section{Analyse et présentation des résultats}

L'analyse des résultats du volet quantitatif a pris en compte la stratégie d'échantillonnage (stratifications, degrés, poids de sondage) et porte sur la population des usagers de drogues pris en charge dans les dispositifs spécialisés et chez les médecins généralistes dans les cinq villes. Un poids de sondage a été affecté à chaque individu en utilisant la méthode généralisée du partage des poids $[8]^{4}$. Les analyses statistiques ont été réalisées avec le logiciel Stata.V.8.2. En analyse univariée, les comparaisons des caractéristiques sociodémographiques et des pratiques entre les hommes et les femmes ont été effectuées avec le test du chi2, avec un seuil à 0,05.

Pour certaines variables, l'analyse porte sur l'ensemble de la population. Les résultats de la comparaison entre hommes et femmes ne sont présentés que si les différences sont significatives. L'ensemble des différences présentées ici étant significatives, ce seuil n'est pas spécifié dans le texte. Pour faciliter sa lecture, les pourcentages seront présentés de manière uniformisee : tout d'abord, les femmes versus les hommes entre parenthèses. Dans les tableaux, les données sont pondérées et les effectifs totaux femmes versus hommes peuvent changer d'une question à l'autre, en raison des non-réponses et des filtres présents dans le questionnaire.

Pour le volet socio-anthropologique, quelques trajectoires de femmes (en encadrés 1 et 2, ou en extraits d'entretiens thématiques) ont été choisies, soit parce que celles-ci illustraient les résultats épidémiologiques, soit parce qu'elles venaient apporter des éléments nouveaux non mis en évidence dans le volet épidémiologique. Ces deux encadrés viennent illustrer de manière " archétypale " des trajectoires de vie chaotiques et la dépendance au conjoint. Afin de garantir l'anonymat et la confidentialité des données, les prénoms des personnes interviewées ont été modifiés ainsi que les noms de certaines villes où elles ont pu séjourner à un moment ou un autre de leur parcours. Une première analyse de contenu thématique a été réalisée à partir d'un travail de codification et d'indexation des entretiens facilité par le logiciel NVivo. II s'agissait ici principalement de partir de l'expérience vécue par les usagers de drogues et de réintégrer les notions de temporalité et de contexte social dans l'analyse des données.

\section{Résultats des deux volets de l'enquête Anrs-Coquelicot}

Les résultats de l'étude épidémiologique menée sur l'ensemble de la population sont disponibles dans un Bulletin épidémiologique hebdomadaire [9]. Dans ce volet épidémiologique, sur 2389 usagers de drogues à qui il a été proposé de participer, 1462 ont accepté, soit un taux de participation de $61 \%$. La population des usagers de drogues est essentiellement masculine (336 femmes soit $26 \%$ et 1125 hommes soit $74 \%$ ), l'âge moyen est de 35,6 ans pour les hommes et de 34,5 ans pour les femmes. Le sexe n'a pas été renseigné pour un seul questionnaire. La

Le calcul des poids de sondage prend en compte les fréquentations multiples de certains usagers de drogues dans les structures d'accueil au cours de la période d'enquête. Plusieurs questions qui leur ont été posées concernant les fréquences de visites au cours de l'enquête dans les différents services ont permis de considérer la méthode généralisée du partage des poids (Lavallée, 2002), qui offre un cadre théorique pour le calcul des poids. 
population a été recrutée pour $58 \%$ dans les dispositifs basseuil ${ }^{5}$ (programmes d'échange de seringues, équipes de rue, ...), $31 \%$ dans les dispositifs haut-seuil (Centres de soins spécialisés pour toxicomanes, ...) et $11 \%$ chez les médecins généralistes. Parmi les non-répondants, $93 \%$ ont été contactés dans les dispositifs spécialisés et $7 \%$ chez les médecins. II n'existe pas de différences significatives entre répondants et non-répondants en ce qui concerne l'âge et le sexe.

Dans l'étude qualitative, 87 entretiens ont été réalisés, dont 30 avec des femmes et 57 avec des hommes. Les femmes représentent donc un tiers de l'échantillon. La moyenne d'âge est de 25 ans pour les femmes et de 30 ans pour les hommes. Les usagers de drogues étaient ici recrutés hors structure au moment de l'enquête pour les trois quart d'entre eux.

\section{Trajectoires de vie}

\section{Ruptures de vie à l'adolescence}

Les trajectoires des usagers de drogues sont souvent chaotiques, scandées par des ruptures de vie dans l'enfance, l'adolescence et à l'âge adulte. Ainsi, globalement, $22 \%$ des usagers de drogues ont déclaré qu'à 16 ans, ils ne vivaient déjà plus chez un membre de leur famille et $73 \%$ affirmaient qu'ils avaient déjà dormi au moins une fois dans la rue depuis l'âge de 18 ans en raison de l'absence de domicile personnel.

Les femmes ont des trajectoires encore moins linéaires que celles des hommes puisque dès l'âge de 16 ans, elles sont proportionnellement plus nombreuses à ne plus vivre dans leur famille (28\% vs $17 \%$ ), c'est-à-dire qu'au moment de l'adolescence, un tiers d'entre elles étaient livrées à elles-mêmes, en fugue, ou placées dans des foyers de la DDASS ou des familles d'accueil (tableau 1).

Les résultats de l'étude qualitative viennent compléter ces chiffres et montrent que durant l'enfance et l'adolescence, les femmes usagères de drogues interrogées sont fréquemment victimes de violences verbales, physiques et d'abus sexuels au sein de la famille, comme l'illustrent les récits de vie présentés en encadrés et dans les citations ci-après. La moitié d'entre elles a évoqué avoir subi ce type de violences. Durant l'enfance et l'adolescence, ces violences ont pu être exercées à leur encontre, mais plus encore envers une autre figure féminine de la famille, telle leur mère. Dans les entretiens, les hommes déclarent également avoir connu des épisodes de violence familiale, mais ils les évoquent moins spontanément, de manière moins détaillée et les présentent de manière moins " traumatique " que les femmes. De plus, seules les femmes rapportent avoir subi des agressions à caractère sexuel. Les différentes postures d'énonciation masculines et féminines des épisodes de violence familiale peuvent être interprétées comme une difficulté à " se laisser aller " à

\footnotetext{
Abaisser le seuil d'exigence revient à ne plus imposer le sevrage comme condition d'accès au dispositif de prise en charge, de soins et de prévention.
}

Tableau 1

Caractéristiques sociodémographiques. Comparaison entre les hommes et les femmes ayant répondu à l'enquête Anrs-Coquelicot 2004, France

\begin{tabular}{lcrrrr}
\hline & $\begin{array}{c}\text { Femmes } \\
\text { n total }\end{array}$ & $\begin{array}{c}\% \\
\star \star\end{array}$ & $\begin{array}{c}\text { Hommes } \\
\text { n total }\end{array}$ & $\begin{array}{c}\% \\
\star \star\end{array}$ & p \\
\hline Vit en couple & 334 & 47,0 & 1120 & 22,9 & 0,000 \\
A des enfants & 336 & 60,5 & 1120 & 46,7 & 0,03 \\
Vit seul & 317 & 38,4 & 1085 & 60,0 & 0,006 \\
Antécédent d'incarcération & 336 & 39,3 & 1122 & 68,7 & 0,000 \\
Ressources (allocations) & 329 & 15,4 & 1102 & 2,3 & 0,003 \\
$\begin{array}{l}\text { Pension alimentaire } \\
\text { Vit chez un membre }\end{array}$ & 329 & 1,3 & 1102 & 0 & 0,000 \\
de la famille à 16 ans & 336 & 71,7 & 1125 & 82,5 & 0,05 \\
\hline
\end{tabular}

- Le n total correspond au nombre de personnes ayant rèpondu à la question.

* Les effectifs ne sont pas présentés car les données sont pondérées.

dévoiler des fragilités personnelles. II peut s'agir ici d'une volonté plus marquée chez les hommes de " sauver la face " face à l'enquêteur, la "fragilité " étant ici plus ou moins consciemment intégrée chez les enquêtés comme une attitude plus féminine. L'intérêt du volet qualitatif prend tout son sens ici en allant audelà de la quantification des événements et en se centrant plutôt sur la compréhension de leur contexte et de leur impact sur des inflexions de trajectoires.

Pour ces femmes, les errances et l'entrée dans la consommation de produits illicites sont d'ailleurs parfois présentées comme un moyen de fuir les violences subies, comme l'évoque Angélique 19 ans : "C'est vrai qu'après, j'ai commencé à faire des fugues et tout ça parce que j'étais en foyer quand-même parce que mon père, il m'a tapé quand-même. Comme il y avait beaucoup d'engueulades et tout ça, ça a été quand-même très loin, et là quand-même je me piquais déjà. »

Comme pour Pierrette 31 ans, la violence au sein de la sphère familiale peut être évoquée a posteriori comme un motif, voire une justification à l'initiation puis à « l'ancrage » dans l'usage de produits : " J'ai 31 ans et le problème c'est que j'ai vécu assez de douleur chez mes parents et tout, je me suis fait taper dessus très jeune chez ma mère, battue plutôt. Mon père, il venait là où j'étais dans la baraque, il me tenait et tout. À un moment, à l'âge de 13 ans, j'en pouvais plus alors je me suis cassée, j'ai fugué, j'en avais marre de me faire frapper dessus. Et après j'ai commencé à fumer, le shit d'abord, déjà en sortant des collèges et tout ça, je fuguais du collège et puis bon je fumais du shit et après j'ai commencé à un peu sniffer de l'héro, du speed et après je me suis mise à l'orténal. ”

Le discours des femmes sur la période de rupture des liens avec la famille à l'adolescence peut s'inscrire dans d'autres logiques argumentatives. Ainsi, pour certaines femmes, l'adoption d'un mode de vie marginal, même s'il s'inscrit dans un contexte d'initiation aux drogues, peut ainsi parfois être interprété comme une forme de libération, à l'instar de Joséphine 24 ans : « En fait, moi 
j'habitais toute seule sur Marseille à partir de 17 ans. J'ai été en foyer, ensuite je me suis fait virer du foyer et à partir de ce moment-là je me suis fait récupérer par le punk en question, qui m'a dit "écoute-moi j'habite dans un squat avec plusieurs personnes, si tu veux, je t'héberge ", voilà comment ça s'est passé. Donc voilà après, il m'a hébergée, au fur et à mesure je suis restée dormir. Pas du tout envie de bouger, j'étais très bien où j'étais, ça sentait la liberté à plein nez ».

L'éloignement de la famille se confond souvent avec l'initiation à la sexualité et aux drogues et marque le point de départ d'une dépendance au conjoint pour l'approvisionnement en produits.

\section{Encadré 1 : Ruptures de vie et initiation aux drogues}

Sun est une femme dont l'enfance et l'adolescence sont ponctuées par des événements traumatiques. Enfant, la jeune femme, aujourd'hui âgée de 29 ans, vit, ainsi que tout le reste de sa famille, dans un climat de terreur orchestré par son père qui les tyrannise et qui fait des punitions corporelles la base de son éducation et de ses rapports conjugaux. L'atmosphère familiale n'est faite que de peur et de violence. Sun garde de cette période le souvenir de sa mère, en sang, s'interposant entre le père et ses enfants pour les protéger, celui de sa sœur hospitalisée, la nuque brisée, et d'elle, multipliant les occasions pouvant éventuellement favoriser son retrait de la famille. Elle vole dans les magasins, à plusieurs reprises, avec l'espoir d'être placée en foyer d'accueil. Sans succès. Elle en arrive à boire des liquides toxiques, ce qui va lui valoir des brûlures de l'œsophage et une hospitalisation, mais pas de placement. C'est dans ce contexte que prend place son initiation aux drogues.

\section{Dépendances vis-à-vis du conjoint}

S'il n'existe pas de différences significatives entre le taux d'activité professionnelle des hommes et des femmes, la majorité est exclue du monde dutravail, car $75 \%$ des usagers de droguesne travaillent pas. Les femmes se distinguent par la source de leurs ressources, qui sont plus souvent issues d'allocations familiales ( $15 \%$ versus $2 \%$ ) ou de pensions alimentaires ( $1 \%$ versus 0 ), ce qui rend leur situation sociale plus fragile car dépendant de l'extérieur. À l'âge adulte, au moment de l'enquête, elles vivent plus souvent en couple ( $47 \%$ versus $23 \%$ ), ont plus souvent des enfants, vivant ou non avec elles, ( $60 \%$ versus $47 \%$ ) et vivent moins souvent seules que les hommes (38\% versus $60 \%$ ) (tableau 1 ).

$\mathrm{Si}$ la situation des femmes (plus souvent en couple avec enfants) donne l'impression d'un mode de vie plus en adéquation avec les conventions sociales dominantes, elle peut également constituer un frein à l'insertion sociale. En effet, dans les entretiens, les femmes usagères de drogues décrivent fréquemment une situation de dépendance vis-à-vis de leur conjoint, en particulier pour l'achat de produits psychoactifs, à l'instar d'Assiatou, 26 ans : "Et donc j'ai continué et puis donc après je suis passée à autre chose, $j$ 'ai rencontré un garçon qui dealait de tout, donc y'avait tout le temps de tout, donc j'étais à fond tout le temps, de tout, donc ça a duré trois ans, et jusqu'au jour où j'ai ouvert les yeux un peu parce que j'étais pas dans la réalité, moi je me voyais bien, même si au fond de moi je savais que c'était pas une solution quoi, ben jusqu'au jour où je me suis aperçue qu'en fait j'allais pas bien du tout, j'avais perdu 15 kilos, j'étais grise, j'avais plus aucune vie sociale. "Quand les rôles de dealer et de partenaire sexuel se confondent, l'accès au produit est facilité au quotidien. Cette confusion des rôles peut alors être vécue comme la cause d'une escalade dans la consommation, puis d'un enfermement dans la dépendance.

L'absence d'autonomie financière et la dépendance vis-à-vis du conjoint peuvent amener certaines de ces femmes à supporter des violences répétées de la part de leur compagnon, comme essaie de le justifierEdwige 35 ans : « Le jour des deux mois de notre fils, le jour des deux mois de $M$, on s'est disputés, c'était le soir et je suis passée par la fenêtre de chez sa mère, je suis tombée de quatre étages. Mais après, j'ai su que c'était lui qui m'avait jetée (...) Je n'ai pas porté plainte parce qu'encore une fois, c'est lui qui venait me voir tous les jours à I'hôpital, il m'apportait ma came, je continuais à tourner à I'héro (...) Et puis quand ils ont commencé à diminuer les doses de Tranxène, tout de suite, j'ai voulu de la came. Et vu que le père de $M$ venait me voir tous les jours, je me shootais à l'hôpital. Tous les jours, il me faisait ma piqûre. Et non, je ne l'ai jamais quitté parce que quand-même, cet amour, c'était violent, mais passionnel, une espèce de relation complètement délirante. Ily y avaiteu beaucoup de passion. Après l'amour était blessé forcément par toutes les blessures qu'il m'avait infligées. Mais en même temps, je dépendais de lui pour la came (...) tu n'as pas une thune en poche, t'es en manque. Je ne tenais pas et toujours je finissais par revenir vers lui. "

À l'inverse, certaines femmes comme Zhiyu (21 ans) ne présentent pas cet accès facilité aux produits par l'intermédiaire des hommes comme une dépendance subie mais plutôt comme une stratégie délibérée : "Et comme j'étais une fille, que j'avais une visuelle plus grande, j'attirais toute l'attention, par rapport à mon physique, c'était facile de trouver les produits aussi parce que quand j'étais avec quelqu'un, s'il avait des produits, j'arrivais à récupérer de lui. ».

De plus, cet accès privilégié aux produits par le biais du conjoint peut également constituer un facteur de protection des femmes vis-à-vis des autres conduites délictueuses (vols, deal) plus souvent observées chez les hommes. Les femmes usagères de drogues ont d'ailleurs moins souvent connu un épisode d'incarcération que les hommes dans leur vie (39\% versus $69 \%$ ) d'après les résultats de l'étude quantitative. Comme nous le verrons plus loin, les femmes ont accès aux drogues plus souvent que les hommes par le biais de la prostitution qui peut s'exercer sous différents registres.

\section{Rapport à la santé}

Pour ce qui concerne les problèmes de santé rencontrés dans les six derniers mois, si les niveaux d'appréciation de leur état de santé sont similaires chez les femmes et les hommes (plus 


\section{Encadré 2 : La dépendance au sein du couple}

Edwige, âgée de 35 ans, a eu une enfance chaotique partagée entre la France et l'Afrique. À la suite du divorce de ses parents, elle est à la charge de son père jusqu'au décès de ce dernier, victime d'un cancer. Elle rejoint alors sa mère et son beau-père en Afrique où elle vit une existence agréable et confortable jusqu'à l'accident de voiture dont est victime son beau-père qui devient alors paraplégique. Suite à son rapatriement en France, l'enfant se retrouve dans une petite bourgade isolée en compagnie d'un beau-père handicapé et d'une mère distante, parfois violente (physiquement et psychologiquement).

Dans un désir de fuir l'existence qu'elle mène, Edwige découvre, alors qu'elle n'a même pas 12 ans, les boîtes de nuits et l'univers qui les entoure parfois : alcool, cannabis, trips. Elle a également son premier rapport sexuel avec un homme majeur. Les rapports conflictuels qu'elle entretient avec sa mère s'aggravent tout au long de son adolescence jusqu'à ce qu'elle soit chassée du domicile familial. Elle a 17 ans. Son départ signe l'arrêt de ses études et le début d'une période d'errance. Sans domicile, coupée de sa famille, la jeune femme rencontre un homme dont elle tombe amoureuse. Héroïnomane, il l'initie à l'héroïne en intraveineuse. Elle vit alors à ses côtés une existence, qu'elle qualifie d'insouciante, marquée par le partage de seringues, des rapports sexuels non protégés, et de consommations excessives. Elle contracte les hépatites B et C.

Convaincue que son compagnon a un avenir professionnel assuré (accessoirement, il deale de l'héroïne), Edwige se réjouit quand elle apprend qu'elle est enceinte. Elle se surprend alors à rêver d'une vie de famille. Le rêve s'effondre quand elle découvre qu'en réalité, son conjoint est un exdétenu, financièrement dépendant de ses parents. Abondamment fournie en produit par son compagnon, la jeune femme s'injecte tout au long de sa grossesse et accouche d'un bébé en manque. Elle est également fréquemment victime de violences qui lui valent plusieurs séjours hospitaliers, des semaines de coma, et de nombreuses cicatrices. Son enfant lui est alors retiré et est confié à sa belle-mère. Les vols qu'elle commet pour subvenir à ses besoins ne suffisent pas, elle reste dépendante à tout point de vue : de son conjoint pour l'argent et les produits, de sa belle-mère pour son enfant. Démunie, elle se tourne vers sa mère, qui la rejette une nouvelle fois.

de $50 \%$ d'entre eux estiment que leur état de santé est plutôt bon et $10 \%$ très bon), certains troubles sont cependant plus fréquemment rapportés par les femmes. Ainsi, les femmes rapportent plus souvent des gonflements des mains et des pieds ( $29 \%$ versus $16 \%$ ) et des troubles oculaires ( $40 \%$ versus $26 \%$ ) (tableau 2). Aucun problème de santé, en revanche, n'est répertorié comme étant plus important chez les hommes.

Les femmes usagères de drogues ont un meilleur niveau de dépistage dans la vie tant pour le VIH (99\% versus $94 \%$ ) que pour le VHC (98\% versus $89 \%$ ). La proportion de femmes ayant effectué un dépistage simultané pour les deux virus est encore supérieure à celle des hommes (98\% versus $88 \%$ ) (tableau 2).

Globalement, comme dans la population générale, ces résultats montrent que les femmes semblent plus à l'écoute de leurs

Tableau 2

Etat de santé et dépistage du VIH et du VHC. Comparaison entre les hommes et les femmes ayant répondu à l'enquête Anrs-Coquelicot 2004, France

\begin{tabular}{lccccc}
\hline & $\begin{array}{c}\text { Femmes } \\
\text { n total }\end{array}$ & $\begin{array}{c}\% \\
\star \star\end{array}$ & $\begin{array}{c}\text { Hommes } \\
\text { n total* }\end{array}$ & $\begin{array}{c}\% \\
\star \star\end{array}$ & p \\
\hline $\begin{array}{l}\text { Gonflements mains/pieds } \\
\text { dans les 6 derniers mois }\end{array}$ & 336 & 28,6 & 1125 & 15,6 & 0,008 \\
$\begin{array}{l}\text { Troubles oculaires } \\
\text { dans les 6 derniers mois }\end{array}$ & 336 & 40,1 & 1125 & 26,1 & 0,04 \\
$\begin{array}{l}\text { Dépistage du VIH dans la vie } \\
\text { Dépistage du VHC dans la vie } \\
\text { Dépistage du VIH }\end{array}$ & 333 & 99,2 & 1120 & 94,1 & 0,000 \\
et du VHC dans la vie & 323 & 98,2 & 1092 & 89,1 & 0,000 \\
\hline
\end{tabular}

* Le $n$ total correspond au nombre de personnes ayant répondu à la question.

* Les effectifs ne sont pas présentés car les données sont pondérées. symptômes et adoptent plus fréquemment des attitudes préventives que les hommes $[10,11]$. Toutefois, ce meilleur niveau de dépistage dans la vie peut être expliqué soit parce que les femmes prennent globalement davantage soin de leur santé que les hommes, soit parce qu'elles s'exposent plus au risque et ont donc plus de raisons " objectives » de se faire dépister.

\section{Usage de drogues et prises de risques}

\section{Des produits spécifiques : crack et sulfates de morphine}

Concernant les produits consommés dans le dernier mois, les femmes se distinguent par une consommation plus importante de crack/free base ( $42 \%$ versus $25 \%$ ), de sulfates de morphine $(20 \%$ versus $10 \%)$ et de solvants $(2 \%$ versus $0,3 \%)$. En revanche, elles consomment moins, de stimulants $(0,4 \%$ versus $4 \%$ ), d'amphétamines ( $3 \%$ versus $8 \%$ ) et d'hallucinogènes ( $1 \%$ versus $4 \%$ ) que les hommes. La consommation quotidienne d'alcool est moins fréquente chez les femmes ( $21 \%$ versus $29 \%$ ) (tableau 3 ).

\section{Une initiation aux drogues et à l'injection avec le partenaire sexuel}

Globalement, la majorité des usagers de drogues ( $83 \%$ ) déclare que leur première injection a été réalisée par un autre usager de drogues. Le contexte de l'initiation et le profil de l'initiateur ont été explorés dans l'approche socio-anthropologique. Ainsi, dans les entretiens, aucun homme n'a évoqué avoir été initié aux drogues par son partenaire sexuel. Les rares usagers masculins initiés par des femmes l'ont été dans le cadre d'une initiation collective au sein d'un groupe de pairs. Chez les femmes, en 
Trajectoires de vie et prises de risques chez les femmes usagères de drogues

revanche, l'initiation aux produits, et en particulier à l'injection, s'effectue généralement dans le cadre de relations amoureuses par le biais du partenaire sexuel, comme le raconte Zhiyu 21 ans : " II y avait un gars que je ne voulais pas voir au début parce qu'il prenait la came, mais quand je cherchais ça, quand je voulais trouver ce produit et je savais que je pouvais en prendre chez lui, par exemple, je me suis mise avec lui parce qu'il voulait être avec moi, mais moi, je ressentais rien, juste pour avoir ça, je me suis mise avec lui, pour commencer, pour connaitre. Et c'est lui qui m'a montré comment shooter. ”

Si pour Zhiyu, la consommation par l'intermédiaire du partenaire sexuel est évoquée comme une opportunité, Violette, 32 ans, présente son initiation à l'injection par le biais de son partenaire sexuel comme une décision personnelle et un moyen d'apprentissage de la pratique d'injection : " La première fois que j'ai touché, c'était un garçon qui me plaisait. Je savais qu'il était séropositif, je savais qu'il se droguait et tout. Eh ben, je m'en suis foutue. Je disais : il me le faut... J'ai commencé à discuter avec lui. Après je suis sortie avec lui, toujours en faisant attention. Je suis sortie avec lui et, un jour, il est allé toucher, il m'a dit : t'as déjà touché ? Moi j'avais dit que oui alors que j'avais jamais touché. II m'a préparé ma seringue et tout, j'avais un peu peur, mais j'avais sniffé une fois et ça m'avait rien fait. Une fois j'avais essayé de sniffer, deux fois même, avec des copines, ça m'avait rien fait du tout. Là, la troisième fois que j'ai rencontré ce garçon, il m'a préparé ma seringue toute neuve et tout. Moi j'étais derrière, eux deux ils étaient devant, c'étaient deux frères, dans la voiture. C'est là, la première fois que je me suis piquée toute seule. ”

Dans certains cas, comme pour Edwige, 35 ans, l'initiation aux drogues par le partenaire sexuel est interprétée a posteriori comme une forme de manipulation en vue d'obtenir des faveurs sexuelles plus facilement : "Avant, le shoot, ça ne m'attirait pas, jusqu'au moment où je me suis sentie tellement paumée que je n'avais plus de limite, plus de barrière. Et puis ce mec-là je le trouvais vachement attirant, c'était la première fois que je rencontrais un mec plus grand que moi, il était métis, beau gosse, du charme, en plus quand je l'ai connu, il était en costard cravate, il travaillait (...) oui je le voyais, il avait l'air bien. Et puis le beau mec que je te décris en fait c'était une belle crapule, il n'en avait rien à faire de me faire mon premier shoot, au contraire peutêtre que pour lui c'était plus simple si j'étais défoncée. Moi je continuais à fumer de l'héro et puis il m'a dit : " attends, ça sera tellement meilleur ", et voilà, il m'a fait ma première piqûre et puis c'est géant parce que c'est super bon. ”

\section{Moins d'injection, mais plus de partage}

Les femmes ont globalement moins recours à l'injection dans la vie $(59 \%$ versus $74 \%)$. En revanche, il n'existe pas de différences significatives concernant la pratique d'injection récente (dans le dernier mois), qui concerne globalement $40 \%$ des usagers de drogues. Parmi ceux qui ont eu recours à l'injection dans
Tableau 3

Consommations de produits psychoactifs et pratiques à risque. Comparaison entre les hommes et les femmes ayant répondu à l'enquête Anrs-Coquelicot 2004, France

\begin{tabular}{|c|c|c|c|c|c|}
\hline & $\begin{array}{l}\text { Femmes } \\
\mathrm{n} \text { total* }\end{array}$ & $\begin{array}{l}\% \\
* *\end{array}$ & $\begin{array}{l}\text { Hommes } \\
\mathrm{n} \text { total}^{*}\end{array}$ & $\begin{array}{l}\% \\
\star \star\end{array}$ & $\mathrm{p}$ \\
\hline $\begin{array}{l}\text { Injection dans la vie } \\
\text { Consommation de crack }\end{array}$ & 336 & 59,2 & 1123 & 73,9 & 0,02 \\
\hline $\begin{array}{l}\text { dans le dernier mois } \\
\text { Consommation de su }\end{array}$ & 336 & 41,8 & 1125 & 25,3 & 0,02 \\
\hline $\begin{array}{l}\text { morphine dans le dernier mois } \\
\text { Consommation de solvants }\end{array}$ & 336 & 20,4 & 1125 & 10,2 & 0,01 \\
\hline $\begin{array}{l}\text { dans le dernier mois } \\
\text { Consommation de stimulants }\end{array}$ & 336 & 2,5 & 1125 & 0,3 & 0,000 \\
\hline $\begin{array}{l}\text { dans le dernier mois } \\
\text { Consommation d'amphétamines }\end{array}$ & 336 & 0,4 & 1125 & 4,1 & 0,000 \\
\hline $\begin{array}{l}\text { dans le dernier mois } \\
\text { Consommation d'hallucinogènes }\end{array}$ & 336 & 2,9 & 1125 & 7,9 & 0,001 \\
\hline $\begin{array}{l}\text { dans le dernier mois } \\
\text { Consommation quotidienne }\end{array}$ & 336 & 0,9 & 1125 & 4,5 & 0,005 \\
\hline $\begin{array}{l}\text { d'alcool } \\
\text { Partage d }\end{array}$ & 336 & 20,8 & 1125 & 29,2 & 0,02 \\
\hline dans & 77 & 254 & 309 & 10,6 & 0,02 \\
\hline cipient, cuiller & 75 & 22 & 2 & 5,5 & 0,000 \\
\hline Partage filtre & 74 & 26,2 & 291 & 4,2 & 0,03 \\
\hline $\begin{array}{l}\text { Don de seringue usagée } \\
\text { à un ami }\end{array}$ & & & & & \\
\hline $\begin{array}{l}\text { à un ami } \\
\text { Injection avec la seringue }\end{array}$ & 75 & 18,3 & 2 & & ग \\
\hline d'un ami & 74 & 0,6 & 293 & 3,2 & 0,03 \\
\hline
\end{tabular}

- Le n total correspond au nombre de personnes ayant répondu à la question.

* Les effectifs ne sont pas prèsentés, car les données sont pondérées.

le dernier mois, les femmes sont plus nombreuses à s'engager dans des prises de risques liées à l'injection. Elles déclarent avoir partagé leur seringue dans le dernier mois plus souvent $(25 \%$ versus $11 \%$ ), ainsi que le " récipient " de préparation du produit ( $22 \%$ versus $5 \%$ ) ou encore le filtre ( $26 \%$ versus $4 \%$ ). Concernant le contexte du partage de seringues, les femmes sont plus nombreuses à déclarer donner une seringue usagée à un ami (18\% versus $4 \%$ ) (tableau 3 ).

Les entretiens font apparaitre que les pratiques de partage du matériel lié à la consommation de drogues (seringue, cuiller, filtre ${ }^{6}$ ) chez les femmes se déroulent le plus souvent au sein du couple. Edwige 35 ans, par exemple, évoque le partage de la seringue au sein du couple, justifié en soi par la proximité conjugale: "C'était en 1991, 90, on entendait parler du sida, mais bon, dans ta tête tu te dis que tu t'en fous, t'es franchement suicidaire, $j$ 'en avais rien à foutre si c'était la même pompe, je n'en ai même pas parlé avec lui, ça ne le dérangeait pas. On était déjà ensemble donc pas de soucis (...) Pas du tout, aucune protection, aucune prudence."

Le filtre est utilisé pour filtrer le produit aspiré dans le récipient (cuiller) avec la seringue. II s'agit genèralement d'un coton, d'un filtre de cigarette ou du filtre stérile disponible dans les steribox. 
Les femmes étant plus dépendantes des autres usagers, et en particulier de leur conjoint pour l'obtention de produit, la possibilité de consommer est étroitement liée aux occasions offertes par les autres consommateurs. Cette absence de maîtrise des circonstances de l'acte de consommer constitue un frein pour avoir à sa disposition du matériel d'injection stérile et favorise ainsi l'exposition au risque vis-à-vis du VIH et des hépatites. L'importance du partage du filtre symbolise le manque d'autonomie des femmes dans leur gestion du produit, les filtres abandonnés par les autres usagers étant un moyen de consommer des résidus de produit de manière économique.

\section{Une vulnérabilité importante face au risque sexuel}

\section{Des partenaires occasionnels plus souvent} usagers de drogues

Les femmes usagères de drogues sont plus nombreuses que les hommes à déclarer avoir eu des relations sexuelles dans les six derniers mois ( $89 \%$ versus $75 \%$ ). La partie du questionnaire relative à la sexualité explorait également le type de partenaire sexuel (stable versus occasionnel) et ses caractéristiques (statut sérologique et rapport aux drogues). Pour ce qui concerne la sexualité avec le partenaire stable, aucune différence significative n'existe entre hommes et femmes, tant pour le rapport aux drogues du partenaire (usager de drogues, usagers de drogues par voie intraveineuse) que pour son statut sérologique vis-àvis du VIH. L'exposition au risque dans le cadre de la sexualité concerne plus particulièrement les usagers de drogues ayant des partenaires occasionnels. Les femmes se distinguent ici. En effet, si elles ne déclarent pas avoir plus souvent des partenaires occasionnels que les hommes, ce partenaire est plus souvent un usager de drogues ( $81 \%$ versus $54 \%$ ) (tableau 4 ).

Dans les entretiens, il apparaît que les figures de partenaire sexuel et de fournisseur principal de produits se confondent, à l'instar de Nour (45 ans) : “ de 1983 à 1986, j'ai rencontré un garçon et en fait, c'était un dealer d'héro donc en fait, on était ensemble parce qu'il y avait l'héro. "(...) " Moi, quand j'étais dans la coke depuis 2001 jusqu'à maintenant, j'ai toujours fait des transactions pour avoir mon bakchich en coke. " Comme cela a été évoqué précédemment, le partenaire sexuel est également celui qui initie aux drogues puis à l'injection et qui approvisionne en produits.

\section{Des relations sexuelles souvent contraintes}

Parmi les usagers de drogues déclarant avoir des partenaires occasionnels, les femmes sont bien plus nombreuses à déclarer avoir des relations sexuelles contre des drogues ( $32 \%$ versus $2 \%$ chez les hommes) ou de l'argent (72\% versus $3 \%$ ). Rapporté à la population globale des usagers de drogues, le recours à la prostitution (considéré comme avoir eu des rapports sexuels contre des drogues ou de l'argent) est également beaucoup plus fréquent chez les femmes, un tiers des femmes $(29 \%)$ déclarent
Tableau 4

Sexualité et prises de risque. Comparaison entre les hommes et les femmes ayant répondu à l'enquête Anrs-Coquelicot 2004, France

\begin{tabular}{lrrrrr}
\hline & $\begin{array}{c}\text { Femmes } \\
\text { n total }\end{array}$ & $\begin{array}{c}\% \\
\star \star\end{array}$ & $\begin{array}{c}\text { Hommes } \\
\text { n total }^{\star}\end{array}$ & $\begin{array}{r}\% \\
\star \star\end{array}$ & p \\
\hline $\begin{array}{l}\text { Relations sexuelles } \\
\text { dans les 6 derniers mois }\end{array}$ & 336 & 89,2 & 1125 & 74,9 & 0,000 \\
$\begin{array}{l}\text { Partenaire occasionnel } \\
\text { usager de drogues }\end{array}$ & 77 & 80,9 & 283 & 58,3 & 0,03 \\
$\begin{array}{l}\text { Sexualité contre drogues } \\
\text { Sexualité contre argent }\end{array}$ & 77 & 32,1 & 283 & 2,1 & 0,000 \\
Prostitution & 77 & 72,5 & 283 & 3,2 & 0,000 \\
\hline
\end{tabular}

- Le n total correspond au nombre de personnes ayant répondu à la question.

* Les effectifs ne sont pas présentés, car les données sont pondérées.

s'être prostituées dans les six derniers mois versus $1 \%$ chez les hommes. Aucune différence significative n'est observée quant au recours au préservatif dans le cadre de la relation stable, de relations occasionnelles ou de la prostitution (tableau 4).

Le volet qualitatif met en évidence que les relations sexuelles des femmes usagères de drogues ne sont pas toujours motivées par un désir partagé et se déroulent souvent dans un contexte de contrainte. Cette contrainte peut, dans une certaine mesure, s'apparenter à différents registres de la prostitution. Ces registres vont des rapports sexuels négociés au sein du couple en échange de produits aux relations tarifées avec des clients pour obtenir l'argent nécessaire à l'achat de produits.

Le premier registre concerne les rapports sexuels qui peuvent se produire au sein du couple avec plus ou moins de contrainte. Ainsi, Pauline, 24 ans, décrit un contexte sexuel constitué de transactions entre drogues et rapports sexuels au sein du couple, marqué par une forme de contrainte latente difficile à identifier et à qualifier: " Je dirais pas que "pour pouvoir consommer il fallait " mais...enfin c'est vrai que des fois, c'est vrai que je me sentais un peu obligée quoi, parce que à l'époque déjà moi j'avais pas...enfin je connaissais pas...enfin je connaissais à peine... J'avais 16-17 ans (...) Et...y a des moments je me souviens où je me sentais un peu obligée. Déjà je dormais chez lui, il m'a hébergée pendant deux semaines...c'était...et puis je fumais à volonté, je sniffais à volonté, enfin tu vois j'étais comme chez moi quoi, j'avais tout quoi. Et...donc sur ce plan là ouais..., enfin quelque part j'avais l'impression que c'était un échange qu'était obligé quoi. »

Pour certaines femmes, l'effet désinhibiteur des substances psychoactives peut être invoqué comme une justification de l'acceptation de la contrainte et atténuer un éventuel sentiment de culpabilité à accepter certaines formes de sexualité, comme l'évoque Angélique 19 ans : " Je me suis retrouvée comme ça, mettons, à être dans des galères, à pas savoir où dormir et tout ça, à aller chez des gens, des connaissances, quand même 
mais... à être hébergée, je me retrouvais chez un type, mettons, hop, il me mettait un ecsta dans la bouche, il croyait que ça allait me faire je sais pas quoi et ça y est, il croyait qu'il allait coucher avec moi et ainsi de suite et ainsi de suite (...) Mais c'est vrai que c'est des effets qu'avec la drogue... c'est vrai que je pense que si j'avais pas pris de drogue, j'aurais pas fait ça. II y a plein de trucs comme ça avec la drogue, j'aurais pas pris de drogue dans ma vie, $j$ 'aurais pas fait plein de trucs comme ça. ”

Le deuxième registre concerne les rapports sexuels tarifés avec des clients extérieurs à la relation de couple. Si le corps des femmes usagères de drogues est encore une fois l'objet de transactions, la négociation avec le partenaire sexuel stable paraît plus claire dans le cas de Nour, 45 ans : «Mais pendant les trois ans que j'étais avec ce copain qui était dealer, en fait il y avait un échange parce que je me prostituais, je lui donnais l'argent de ma prostitution et en échange lui, il me donnait ma came. J'allais pas chercher ailleurs parce qu'en fait, lui, c'était que du bon produit qu'il avait, donc j'allais pas ailleurs, je l'avais à la maison. ”

Ainsi, chez les femmes usagères de drogues, les frontières de la prostitution sont floues, dans la mesure où sexualité et usage de drogues sont étroitement liés et la question de la dépendance au conjoint pour l'obtention de produits se pose fréquemment, comme cela a été mentionné à plusieurs reprises.

\section{Vulnérables au sein de la prostitution}

Dans le contexte de la prostitution, les femmes usagères de drogues se situent dans une posture fragile au moment de la négociation avec le client. Cette vulnérabilité est liée à la nécessité d'apaiser les effets du « manque " lié aux produits qui peut les amener à accepter des tarifs très bas en comparaison des prix du marché, voire à accepter certaines pratiques demandées par les clients comme le non-usage systématique du préservatif. La transaction est alors systématiquement défavorable à l'usagère de drogues, comme l'explique Zhiyu 21 ans: « Il $y$ a des gens qui essaient de profiter de ça, ce sont des gros dealers de merde, on les appelle, ils utilisent ça, mais parfois, j'en ai connu qui n'utilisent même pas. Et ils essaient de trouver des filles qui sont déjà accros à ça pour les baiser facilement contre leur dose. Et surtout, ce qui est malheureux, tu arrives déjà à vendre ton corps, mais c'est pire que faire la pute, tu as un prix fixe à toi, par exemple, tu te dis que pour 200 euros tu le fais. Mais à chaque fois, quand tu le fais pour la dope, ce n'est jamais la quantité que tu mérites. Normalement, un gramme ça fait maximum 50 euros, mais un dosage, s'il te file un truc à 10 euros, t'es ok. C'est encore plus la pitié que de faire la pute, parce que tu vends ton corps à 10 euros, sinon, t'as pas d'autre choix. ”

Dans son récit, Zhiyu 21 ans évoque la contrainte et la fragilité dans lesquelles se situent les femmes usagères de drogues pour consommer : " Je ne sais pas, mais la drogue je peux dire que ça te rend plus violent dans ton caractère, plus vicieux. Parce que, pour récupérer ça, tu cherches des vices, parce que tu ne peux pas récupérer avec des conditions normales : payer et récupérer. Parfois ton argent, c'est pas suffisant et si tu n'a pas de sous, tu ne peux pas te permettre de dire que "c'est pas grave, si je n'ai pas les sous, je n'en prends pas aujourd'hui, ce n'est pas la fin du monde ", c'est pire que la fin du monde. Du coup quand tu n'arrives pas à trouver ce produit, tu es ok pour faire... les gens qui arrivent à vendre leur corps, c'est déjà super honteux pour soi-même. S'ils arrivent à ce niveau-là, je pense que c'est pas facile (...) Et quand tu commences à prendre comme ça, tu arrives à des niveaux où vraiment c'est la honte pour moi, quand tu arrives à un niveau où tu essaies même de vendre ton corps pour récupérer juste une petite trace qui va te rendre mieux. "

Si la prostitution peut au départ constituer un moyen pour se procurer de l'argent nécessaire à la consommation, la relation entre prostitution et consommation peut progressivement se complexifier, les produits devenant un moyen de supporter les relations sexuelles avec des clients. Certaines femmes, à l'instar de Ndeye, 37 ans, évoquent une forme de spirale entre la prostitution et la prise de produits : «Prostitution, c'est pour ça que je me suis mise dedans. Parce que je peux pas le faire à jeun. C'est dur, hein. De donner ton corps, tout ça, c'est difficile. C'est par rapport à ça que j'ai pris, que j'ai commencé la cocaine. occasionnellement. Ça veut dire deux, trois fois par semaine. (La première fois que tu rentres en prison). Pour vol. J'ai toujours fait des vols à l'étalage. Vols et j'ai fait des cambriolages beaucoup. J'ai fait 13 ans en 7 fois (...) C'est après le ras-le-bol de la prison, je voulais plus re-rentrer, c'était en 1998, je me suis mise dans la prostitution. ”

\section{Conclusion}

D'un point de vue méthodologique, dans cet article, une place plus importante est accordée aux données épidémiologiques, mais c'est ici la question de la triangulation des données qui est posée et non pas celle de la supériorité d'une technique d'enquête sur une autre (quantitative ou qualitative). Ces deux approches disciplinaires et méthodologiques ne se substituent pas l'une à l'autre, mais se complètent. L'épidémiologie permet ici de décrire, de mesurer et d'accéder dans une certaine mesure à la généralisation, et la socio-anthropologie, d'approfondir des données et de comprendre des processus sociaux, à partir d'un nombre plus limité de cas [12]. Ces résultats devront être confirmés par une analyse plus approfondie aussi bien des données quantitatives que qualitatives.

Ces premiers résultats de l'enquête Anrs-Coquelicot constituent des pistes de réflexion intéressantes autour de la spécificité des trajectoires des femmes et des prises de risque liées à l'usage de drogues et à la sexualité. La confrontation des données épidémiologiques et socio-anthropologique met en évidence que les femmes usagères de drogues peuvent être qualifiées de "vulnérables parmi les vulnérables " vis-à-vis des risques 
infectieux et en particulier du VIH. Dans le volet socio-anthropologique, il est important de prendre en compte le contexte d'énonciation. Les femmes, en effet, se sont prêtées aux entretiens sans difficulté et elles se sont livrées encore plus facilement que les hommes. Elles ont longuement raconté leurs trajectoires dans le détail et se sont attardées sur les traumatismes subis durant les différentes phases de leur existence. Leurs trajectoires paraissent donc plus archétypales dans la souffrance que celles des hommes, sans qu'il soit évident de distinguer si cette différence est liée à une « réalité éprouvée " par l'individu ou à un " effet de discours ". De plus, il est probable que les biais de désirabilité sociale aient davantage concerné les hommes que les femmes, en particulier sur des aspects liés à la prostitution car chez les hommes, l'activité prostitutionnelle peut renvoyer aux pratiques homosexuelles.

Les résultats issus de notre enquête sont toutefois confortés par ceux de la littérature internationale. Ainsi, les femmes usagères de drogues sont dans une situation de double fragilité dans la sphère de la consommation de produits et dans celle de la sexualité. Cette vulnérabilité est principalement liée à leur dépendance financière vis-à-vis des hommes pour se procurer des drogues. Les femmes usagères de drogues sont plus précaires et moins autonomes socialement, leurs ressources consistant plus souvent en prestations sociales $[13,14]$. Dans la dynamique du couple, le conjoint usager de drogues est souvent le détenteur du produit, l'initiateur aux drogues et à l'injection [15]. Cette dépendance au conjoint expose les femmes au risque de partage du matériel lié à l'injection en raison de leur manque d'autonomie quant au choix des circonstances de consommation. La temporalité de l'acte d'injection qui succède à l'injection du conjoint en utilisant le même matériel expose les femmes de manière importante aux risques de transmission du VIH et du VHC [14].

Tout au long de leurs trajectoires, ces femmes semblent soumises à la contrainte sexuelle à la fois à l'adolescence puis à l'âge adulte. Leur corps est fréquemment l'objet de transaction en échange d'argent ou de drogues. Elles déclarent avoir plus souvent un partenaire sexuel occasionnel usager de drogues: deux tiers des femmes ont des partenaires sexuels usagers de drogues, alors que ce n'est le cas que d'un tiers des hommes [16]. Et les femmes ayant un partenaire usager de drogues sont plus susceptibles de s'engager dans des comportements à risque [6]. Au sein même du monde de la prostitution, les usagères de drogues sont souvent stigmatisées car elles sont accusées par les prostituées « traditionnelles " d'accepter plus facilement des pratiques sexuelles non protégées et souvent à moindre coût [17]. La relation étroite entre consommation de produits et sexualité tend donc à accentuer l'exposition au risque chez les femmes, les effets psychoactifs des produits pouvant diminuer le niveau de vigilance et la dépendance au conjoint, réduisant également leur capacité de négociation quant à la protection des rapports sexuels. Cette vulnérabilité sociale s'ajoute à une vulnérabilité d'ordre biologique, puisque la transmission du VIH est sept à neuf fois plus fréquente dans le cadre de l'exposition homme-femme que femme-homme [18].

De manière globale, les femmes usagères de drogues s'engagent donc plus dans des comportements à risque que les hommes, tant vis-à-vis des drogues que de la sexualité $[2,19]$.

$\mathrm{Au}$ total, les résultats de cette première analyse de l'enquête Anrs-Coquelicot montrent que les femmes usagères de drogues cumulent les vulnérabilités face aux risques de transmission du VIH et du VHC dans les contextes de l'usage de drogues et de la sexualité. La prévention auprès de ces femmes est complexe. Elle doit pouvoir prendre en compte les déterminants biologiques, comportementaux et sociaux des prises de risques et proposer des approches à la fois individuelles et communautaires [20]. II paraît donc important d'intégrer la dimension sociale des rapports hommes/femmes et l'ensemble des formes de contraintes et de violences auxquelles sont soumises les femmes usagères de drogues. L'autonomisation de celles-ci vis-à-vis de leur conjoint usager de drogues et la capacité à négocier la protection au sein du couple lors des rapports sexuels et de la consommation de produits constituent les leviers de la prévention dans cette population.

\section{Remerciements}

A tous les usagers de drogues, les enquêteurs et les professionnels des dispositifs spécialisés et les médecins généralistes ayant participé à l'enquête dans les cinq villes.

Paris : Nova Dona, Pierre Nicolle, Horizons, La Corde Raide, Charonne, SOS DI, Drogues Jeunesses, Moreau de Tours, Murger, Cassini, Emergence, EGO, MDM, AIDES, La Terrasse, Marmottan, Dr Majerloch, Dr Margelisch, Dr Molinier, Dr Joory, Dr Ekue, Dr Bezanson, Dr Demoor, Dr Francoz, Dr Gandour

Lille : Boris Vian, CITD, Espace du Possible, Le Cèdre Bleu, AIDES, Dr Marousez, Dr Heunet, Dr Messaadi, Dr Debomy, Dr Matton, Dr Riff, Dr Flageollet
Strasbourg: Espace Indépendance, ALT, Le fil d'Ariane, Dr Bernard-Henry, Dr Verhnes, Dr Garnier, Dr Gras, Dr Michel, Dr Rolland-Jacquemin, Dr Sellam

Bordeaux : MDM, CEID, Montesquieu, Pellegrin, Parlement Saint-Pierre, Dr Lorans, Dr Quilichini, Dr Sagardoy, Dr Thibaut, Dr De Ducla, Dr Canovas

Marseille: AMPTA, MDM, AIDES, Sainte-Marguerite, Intersecteur des pharmacodépendances, SOS DI, Le Cabanon, Dr Beria, Dr Federici, Dr Philibert, Dr Martin, Dr Robichon, Dr Brun, Dr Hakoun, Dr Hariton, Dr Chiappe

\section{Enquêteurs de l'enquête épidémiologique :}

Paris : A. Aberkane, I. Alet, P. Boudet, B. Burnel, C. Flament, S. Golenishev, E. Guillais, C. Hamelin, H. Léon, L. Quaglia, J. Quenet-Vincent, M. Roussier, M. Tiloy, B. Schuh 
Lille : P. Legroux, A. Luyckx, M. Makouala, C. Sarazin

Strasbourg : M. Duwig, N. Olivier-Martin, J. Oswald,

Bordeaux : F. Bordes, P. Chambreau, E. Lion, E. Martinheira, E. Rappeneau

Marseille : C. Coquillat, J. Denoyer, H. Habert, C. Latard, M. Mounition, H. Sigaud

Partenariat méthodologique de I'Ined : C. Lefevre,M. Quaglia, N. Razandratsima, G. Vivier

\section{Suivi des enquêteurs : K. Guenfoud, A-M. Noel}

Enquêteurs de la recherche socio-anthropologique : A. Benrezhkala, F. Chatot, L. Oudaya, S. Priez

Comité scientifique : F. Beck, V. Doré, A. Ehrenberg, JM. Firdion, I. Grémy, F. Lert

\section{Références bibliographiques}

1. Lejeune C, Simmat Durand L, Gourarier L, Aubisson S. Prospective multicenter observational study of 260 infants born to 259 opiate-dependent mothers on methadone or high-dose buprenorphine substitution. Drug and Alcohol Dependance $2006 ; 82$ (3) : 250-257.

2. Vidal-Trécan G, Coste J, Coeuret M, Delamare N, Varescon Pousson I, Boissonas A. Les comportements à risque des usagers de drogues par voie intraveineuse : les femmes prennent-elles plus de risques de transmission des virus VIH et VHC ? Rev Epidemiol Sante Publique 1998 ; 46 : 193-204.

3. Maranda M, Chenglong H, Rainone G. Crack Cocaine and Sex. Journal of Psychoactive Drugs $2004 ; 36$ (3) : 315-322.

4. Strathdee SA, Galai N, Safaiean M, Celentano DD, Vlahov D, Johnson L, Nelson KE. Sex differences in risk factors for HIV seroconversion among injection drug users : a 10-year perspective. Arch Intern Med 2001 May 28 ; 161(10) : 1281-8.

5. Spittal $P$ et al. Risk factors for elevated HIV incidence rates among female injection drug users in Vancouver. JAMC 2002; 166 (7) : 894-899.

6. Fitzgerald $T$ et al. Factors associated with HIV/AIDS high risk behaviours among female injection drug users. AIDS Care 2007 ; 19 (1) : 67-74.

7. Jauffret-Roustide M. Sciences sociales et épidémiologie: des approches méthodologiques qui se complètent, à propos de la question des pratiques à risque chez les usagers de drogues. BEH $2006 ; 2 / 3: 21-23$.
8. Lavallée P. Le sondage indirect ou la méthode généralisée du partage des poids. Bruxelles : Editions de l'Université de Bruxelles. Ellipses, 2002.

9. Jauffret-Roustide M, Couturier E, Le Strat Y, Barin F, Emmanuelli J, Semaille C et al. Estimation de la séroprévalence du VIH et du VHC et profils des usagers de drogues en France, étude InVS-Anrs Coquelicot, 2004. BEH 2006 ; 33 : 244247.

10. Aiach P, Leclerc A, Philippe A. Facteurs de différenciation dans la déclaration de symptômes. RESP $1981 ; 29: 27-44$.

11. Aliaga C. Les femmes sont plus attentives à leur santé que les hommes. Insee Première $2002 ; n^{\circ} 862$.

12. Jauffret-Roustide M, Emmanuelli J, Barin F, Quaglia, P. Arduin, A. Laporte et al. Impact of a harm reduction policy on HIV and HCV transmission among drug-users. Recent French Data. The Anrs-Coquelicot Study. Substance Use and Misuse 2006 ; 41 (10-12) ; 1603-1622.

13. Hser Y, Anglin M, Booth M. Sex differences in addict careers. 3. Addiction. Am J Drug Alcohol Abuse 1987; 13 : 231-251.

14. Freeman R, Rodriguez G, French J. A comparison of male and female intravenous drug users' risk behaviors for HIV infection. Am J Drug Alcohol Abuse $1994 ; 20$ (2) : 129-157.

15. Kral AH, Blumenthal RN, Erringer EA, Lorvick J, Edlin BR. Risk factors among IDUs who give injections to or receive injections from other drug users. Addiction $1999 ; 94: 675-83$.

16. Rosenbaum M. Women on heroin. New Brunswick, NJ : Rutgers University Press, 1981.

17. Pryen S. Usage de drogues et prostitution de rue. Sociétés Contemporaines $1999 ; 36: 33-51$.

18. Padian NS, Shiboski SG, Glass So, Vittinghoff E. Heterosexual transmission of human immunodeficiency virus (HIV) in northem California : Results from a ten-year study. American Journal of Epidemiology 1997; 146 : 350-357.

19. Evans J, Hahn J, Page-Shafer K, Lum P, Stein E, Davidson P et al. Gender differences in sexual and injection risk behavior among active young injection drug users in San Francisco (the UFO Study). Journal of Urban Health 2003; 80 (1) : $137-146$.

20. Latka M. Drug using women need comprehensive sexual risk reduction interventions. CID 2003; 37 : S445-450. 